\title{
Possible Nagashima-Type Palmoplantar Keratosis in Two Siblings
}

\author{
Kio Park ${ }^{\mathrm{a}} \quad$ Kenji Kabashima $^{\mathrm{b}}$ Yoshiki Miyachi $^{\mathrm{b}}$ \\ ${ }^{a}$ Department of Dermatology, Yamato Takada Municipal Hospital, Nara, and ${ }^{b}$ Department \\ of Dermatology, Kyoto University Graduate School of Medicine, Kyoto, Japan
}

\section{Key Words}

Nagashima-type palmoplantar keratosis $\cdot$ Siblings $\cdot$ Transgressive PPK

\begin{abstract}
Nagashima-type palmoplantar keratosis (PPK) is an autosomal recessive, transgressive and non-progressive form of PPK. It was once described as a mild form of mal de Meleda, but it is now proposed as a novel entity of PPK. Since its pathogenesis remains unclear, it is important to clarify the mode of inheritance. Here, we present a case of possible Nagashima-type PPK in 2 siblings. The siblings had non-affected parents, suggesting that the mode of inheritance was autosomal recessive. At present, reports on PPK in the English literature are limited, and thus the clinical features of Nagashima-type PPK may not be well appreciated in Western countries. More reports and concise clinical observations with genetic studies are required to establish this new entity of PPK.
\end{abstract}

\section{Introduction}

Nagashima-type palmoplantar keratosis (PPK) is characterized by transgressive and non-progressive keratosis with an autosomal recessive trait. Nagashima-type PPK was first described in Japan in 1977 and then followed by more than 20 cases in the Japanese literature [1]. Since Nagashima-type PPK is generally seen in isolated cases, it has been suggested that the mode of inheritance is autosomal recessive. However, a case of Nagashima-type PPK in 2 siblings [2] as well as a familial case of this type of PPK, occurring in father and son [3], was also reported. The latter report raises the question of whether Nagashimatype PPK is mediated only through the autosomal recessive trait. Here, we present another case of 2 siblings with possible Nagashima-type PPK.

Kio Park, MD

Department of Dermatology, Yamato Takada Municipal Hospital

1-1 Isonokitamachi, Yamato Takada

Nara 635-0094 (Japan)

E-Mail kiopark2@gmail.com 
Park et al.: Possible Nagashima-Type Palmoplantar Keratosis in Two Siblings

\section{Case Presentation}

A 9-year-old girl and her 6-year-old sister presented with bilateral reddish, palmoplantar, hyperkeratotic lesions with erythematous borders (fig. 1a-d). The lesions transgressed to the extensor surface of the fingers and toes. These clinical manifestations were already observed within the first 3 years of their lives. They had been treated with betamethasone valerate $0.1 \%$ and gentamicin sulfate ointment, which resulted in no improvement. Their conditions were associated with hyperhidrosis on the palms and soles with distinct odor and maceration. Only the elder sister exhibited the clinical manifestations on her elbows and knees (fig. 1e, f), and both patients showed no involvement of the hair, nails, or teeth. Otherwise, there were no other physical complications. The patients were isolated cases in their family, and their parents were a non-consanguineous couple. The parents declined a skin biopsy.

The clinical manifestation, including transgressive PPK to the extensor surfaces, indicated a diagnosis of Nagashima-type PPK or mal de Meleda (MDM). MDM is an autosomal recessive PPK with a progressive clinical course throughout the patients' lives. Since the patients in this report were still young, it will require more time to determine whether their clinical status is progressive or not. In addition, MDM is usually accompanied by perioral erythema, brachydactyly, and nail abnormalities. Hyperkeratosis extends to the dorsal surfaces of the hands and feet, and the progressive lesions, including constricting bands surrounding the digits, can result in spontaneous amputation. While the mutation in SLURP1 is known to be responsible for MDM [4], genetic analysis has not yet identified the responsible gene in Nagashima-type PPK.

Nagashima-type PPK was proposed as a novel entity of PPK [1], and the manifestations are much less severe and non-progressive after puberty [1]. Although we could not exclude a diagnosis of MDM because the parents declined genetic analysis, the clinical manifestations seen in our patients were consistent with Nagashima-type PPK.

\section{Conclusion}

Herein, we showed a second case of possible Nagashima-type PPK in 2 siblings in the English literature. Our patients had non-affected parents, suggesting that the mode of inheritance was autosomal recessive, though both recessive and dominant inheritance of Nagashima-type PPK may occur [3]. At present, reports on PPK in the English literature are limited, and thus the clinical features of Nagashima-type PPK may not be well appreciated in Western countries. More reports and concise clinical observations with genetic studies are required to establish the new entity of PPK. We believe the accumulation of information on the disease may reveal its underlying pathomechanism in the near future.

\section{References}

1 Kabashima K, Sakabe J, Yamada Y, Tokura Y: 'Nagashima-type' keratosis as a novel entity in the palmoplantar keratoderma category. Arch Dermatol 2008;144:375-379.

2 Isoda H, Kabashima K, Tokura Y: 'Nagashima-type' keratosis palmoplantaris in two siblings. J Eur Acad Dermatol Venereol 2009;23:737-738.

3 Nakamizo S, Takahashi K, Miyachi Y, Kabashima K: A familial case of Nagashima-type palmoplantar keratosis. Eur J Dermatol 2010;20:507-508. 
Park et al.: Possible Nagashima-Type Palmoplantar Keratosis in Two Siblings

Fischer J, Bouadjar B, Heilig R, Huber M, Lefèvre C, Jobard F, Macari F, Bakija-Konsuo A, Ait-Belkacem F, Weissenbach J, Lathrop M, Hohl D, Prud'homme JF: Mutations in the gene encoding SLURP-1 in Mal de Meleda. Hum Mol Genet 2001;10:875-880.
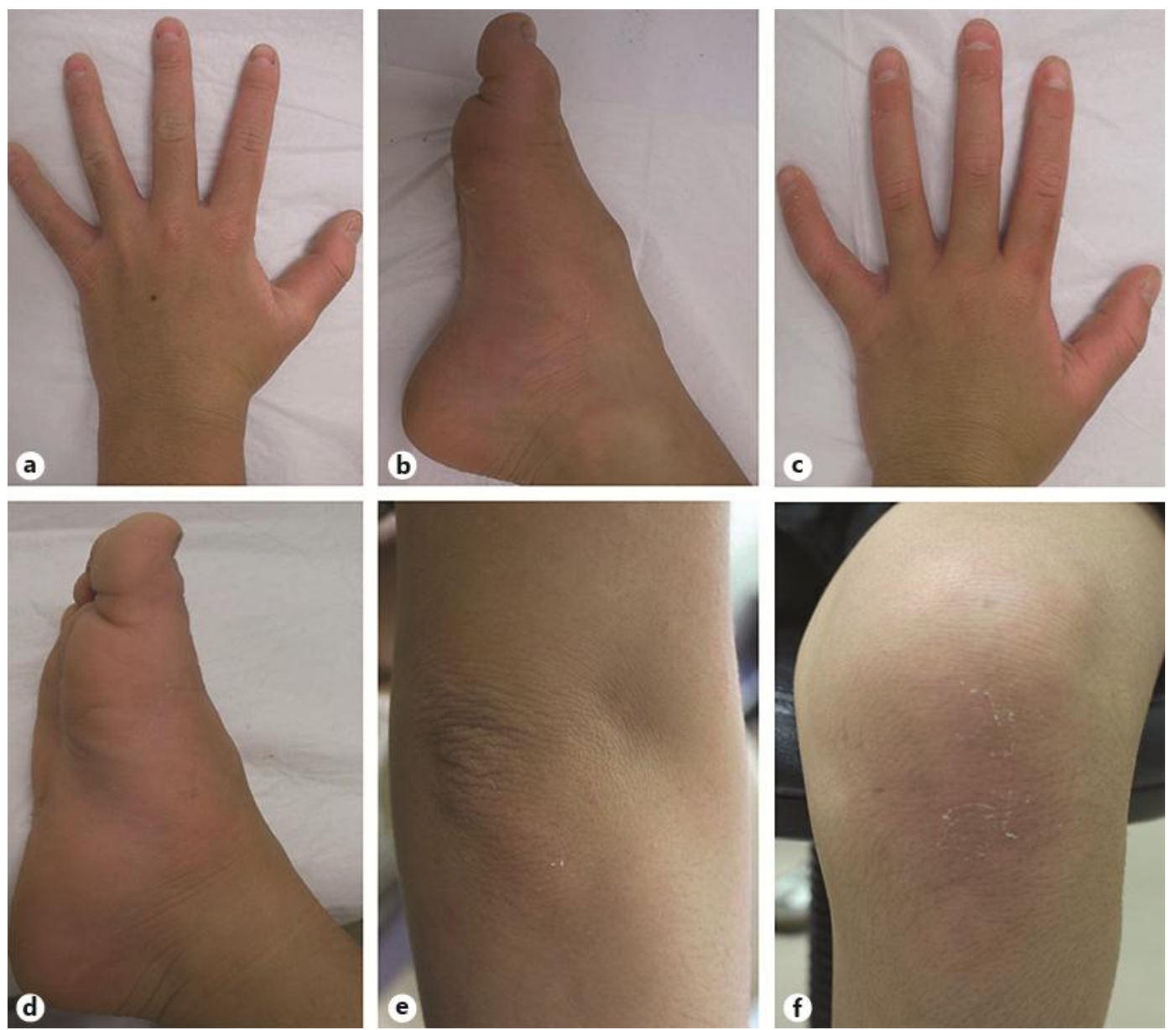

Fig. 1. Clinical appearance of 2 siblings with Nagashima-type PPK. The pictures show bilateral reddish, palmoplantar, hyperkeratotic lesions with erythematous borders of the palms, soles, elbows, and knees (a, b, e, f elder sister; c, d younger sister). 